\title{
Full Angle Dependence of the Four-Loop Cusp Anomalous Dimension in QED
}

\author{
R. Brüser ${ }^{*}$ \\ Theoretische Physik 1, Naturwissenschaftlich-Technische Fakultät, Universität Siegen, 57068 Siegen, Germany \\ C. Dlapa $\odot{ }^{\dagger}$ J. M. Henn $\oplus^{\dagger},{ }^{\dagger}$ and K. Yan $\odot^{\S}$ \\ Max-Planck-Institut für Physik, Werner-Heisenberg-Institut, 80805 München, Germany
}

(Received 20 July 2020; revised 6 October 2020; accepted 14 December 2020; published 11 January 2021)

\begin{abstract}
The angle-dependent cusp anomalous dimension governs divergences coming from soft gluon exchanges between heavy particles, such as top quarks. We focus on the matter-dependent contributions and compute the first truly nonplanar terms. They appear at four loops and are proportional to a quartic Casimir operator in color space. Specializing our general gauge theory result to U(1), we obtain the full QED four-loop angle-dependent cusp anomalous dimension. While more complicated functions appear at intermediate steps, the analytic answer depends only on multiple polylogarithms with singularities at fourth roots of unity. It can be written in terms of four rational structures and contains functions of up to maximal transcendental weight seven. Despite this complexity, we find that numerically the answer is tantalizingly close to the appropriately rescaled one-loop formula, over most of the kinematic range. We take several limits of our analytic result, which serves as a check and allows us to obtain new, powersuppressed terms. In the antiparallel lines limit, which corresponds to production of two massive particles at threshold, we find that the subleading power correction vanishes. Finally, we compute the quartic Casimir contribution for scalars in the loop. Taking into account a supersymmetric decomposition, we derive the first nonplanar corrections to the quark antiquark potential in maximally supersymmetric gauge theory.
\end{abstract}

DOI: 10.1103/PhysRevLett.126.021601

Introduction.-Low-energy gluons lead to large contributions in gauge theory scattering amplitudes and cross sections. The ability to predict and resum these enhanced terms is critical for phenomenology. It has been understood that scattering amplitudes factorize into a processdependent finite part and a universal divergent part [1]. The latter is determined by a set of anomalous dimensions and by the soft anomalous dimension matrix, which describes the renormalization of products of Wilson lines pointing in the particles' directions [2]. A deeper understanding of infrared divergences is critical for developing manifestly finite approaches to cross sections [3] or for deriving quantitative predictions for the finite part of scattering amplitudes from symmetries of the $S$ matrix [4].

Many studies have focused on the planar limit of massless gauge theories, where significant simplifications occur $[5,6]$ and where one may glean insights from integrability [7]. However, nonplanar terms in QCD may be numerically dominant, and finite mass effects are important in many

Published by the American Physical Society under the terms of the Creative Commons Attribution 4.0 International license. Further distribution of this work must maintain attribution to the author(s) and the published article's title, journal citation, and DOI. Funded by SCOAP ${ }^{3}$. situations, for example, when producing a top quark pair near threshold.

The multiparton soft anomalous dimension matrix is known to two loops [8] and exhibits a simple dependence on the scattering angles. Whether or not this continues at higher loops is an open question. We will provide further insights into this issue by exploring the two-line case, which is given by the cusp anomalous dimension.

The cusp anomalous dimension depends on the Euclidean angle $\phi$ formed by the two Wilson lines. It is known to three loops [9-11]. Thanks to non-Abelian exponentiation, it depends on a reduced set of color factors, and truly nonplanar corrections appear for the first time at four loops. They multiply quartic Casimir operators and have recently been computed for the case of massless particles [12-16], thereby settling earlier questions about Casimir scaling. In this Letter, we consider the massive case and determine the matter-dependent quartic Casimir term in QCD. This determines the complete four-loop cusp anomalous dimension in QED.

Our Letter sheds new light on an observed iterative pattern, which allows one to predict matter-dependent terms from lower-loop orders [10,11]. Previous work showed that at four loops this pattern does not hold for all color structures [17]. The term we computed is precisely the one that captures the deviations from the conjecture in 
QED. We compare it quantitatively to the conjectured one, beyond the previously known limits.

We also derive new results in several physically important limits. Interestingly, we find that the first powersuppressed terms in the quark-antiquark threshold limit vanish. Moreover, we determine the first nonplanar correction to the three-loop quark antiquark potential in $\mathcal{N}=4$ super Yang-Mills (SYM).

Methodology.-We compute the vacuum expectation value of the off-shell Wilson lines in momentum space, using the conventions of heavy quark effective theory (HQET) [11]. We consider the gauge group $\mathrm{SU}\left(N_{c}\right)$, see [18] for our conventions, and perform calculations in covariant gauge, which allows us to check gauge invariance of the results. We obtain results for QED by adjusting the QCD color factors according to $C_{A}=d_{R} d_{A} / N_{R}=0$ and $C_{F}=T_{F}=d_{R} d_{F} / N_{R}=1$ [19] and by replacing the strong coupling strength $\alpha_{s}=g_{\mathrm{YM}}^{2} /(4 \pi)$ by the fine structure constant $\alpha$.

We are interested in the matter-dependent quartic Casimir term of the four-loop cusp anomalous dimension,

$$
\left.\Gamma_{\text {cusp }}\right|_{\alpha_{s}^{4}}=\left(\frac{\alpha_{s}}{\pi}\right)^{4} \frac{d_{R} d_{F}}{N_{R}}\left[n_{f} B(x)+n_{s} C(x)\right]+\cdots,
$$

where $B(x)$ and $C(x)$ are the functions we wish to determine, $x=e^{i \phi}$, and where the number of (light) fermions and scalars (canonically coupled to the gauge field) is denoted by $n_{f}$ and $n_{s}$, respectively. The dots stand for other color structures [18], which we do not consider. The Feynman diagrams relevant for the calculation of $B(x)$ are shown in Fig. 1. We evaluate them, and the ones needed for $C(x)$, in dimensional regularization, with $D=4-2 \epsilon$. The cusp anomalous dimension is obtained from the coefficient of the $1 / \epsilon$ pole [18].

We relate the integrals appearing in the Feynman diagrams to a conveniently chosen integral basis [20] and then determine the basis integrals by the method of differential equations (DEs) [21]. In the process, we need to handle large systems of linear equations representing integration by parts identities, which we generate and solve using the codes FIRE6 [22] and LiteRed [23]. For most of the

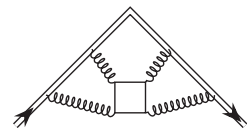

(a)

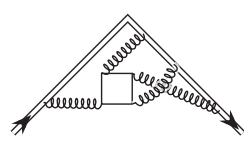

(d)

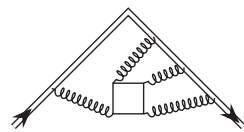

(b)

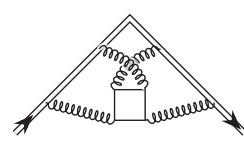

(e)

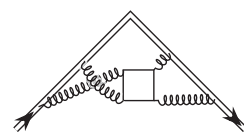

(c)

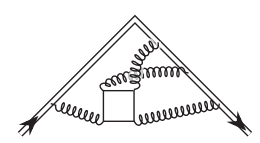

(f)
FIG. 1. Figs. (a)-(f) are Feynman diagrams contributing to the four-loop quartic Casimir term $B$ in Eq. (1). calculations we use FIRE6's finite field methods and reconstruct the full $x$ and $D$ dependence using the techniques of [24] (see also [25]). We reconstruct only the DEs and the Feynman diagrams we need, as opposed to creating integral tables. Thanks to choosing an improved basis, relatively few finite field evaluations are required for this step.

The DEs for the integral families shown in Fig. 1 involve on the order of 500 integrals (needed to describe all integrals sharing the same or fewer propagators), with coupled subsystems of size up to 17 and denominators of up to degree-20 polynomials in $x$ and $D$. We wish to find an integral basis that significantly simplifies the differential equations [20]. To solve this complicated problem in an automated way, we develop further the algorithm of [26] that relies on only a partial knowledge of a canonical basis.

To provide input for the algorithm, we use several ideas: for up to nine propagators, we find candidate uniform weight HQET integrals by a position-space analysis [11], while for integrals with ten or more propagators, we perform a leading singularity analysis with the help of the algorithm [14]. Moreover, we adapt [26] to scan over a larger set of candidate integrals automatically, and to use knowledge of all basis integrals that are known to be uniform weight. Finally, we also make use of the complementary method $[27,28]$. Given the information found by this means, we first transform the diagonal blocks (this is equivalent to considering integrals where propagators are replaced by delta functions) of the DE into canonical form. In a second step, we transform the off-diagonal blocks of the full DEs.

In this way, we obtain a canonical form of the DEs for family (a), (b), (d), and (f),

$$
d \vec{f}(x, \epsilon)=\epsilon \sum_{k} \mathbf{m}_{k}\left[d \log \alpha_{k}(x)\right] \vec{f}(x, \epsilon),
$$

where $\vec{f}$ is the vector of basis integrals for each integral family, $\mathbf{m}_{k}$ are matrices with constant entries, and $\vec{\alpha}=$ $\left\{x, 1+x, 1-x, 1+x^{2}, 1-x+x^{2},[(1-\sqrt{-x}) /(1+\sqrt{-x})]\right.$, $[(1-\sqrt{-x}+x) /(1+\sqrt{-x}+x)]\}$. Setting $x=-z^{2}$, we solve Eq. (2) in terms of multiple polylogarithms [29] to the order in $\epsilon$ needed. The boundary values are taken from [17] or are determined from physical consistency conditions.

For the families (c) and (e), we proceed in the same way for all integrals up to nine propagators and for all diagonal blocks, except for the ten-propagator sector shown in Fig. 2. For this sector we suspect that algebraic basis transformations are insufficient to bring them into canonical form

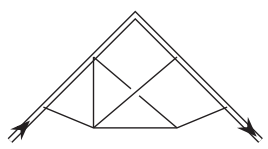

FIG. 2. Integral sector involving special functions that do not appear in the final result. 
(see Supplemental Material [30]). A canonical form can likely be achieved by enlarging the function space [29], but for the scope of this Letter we can proceed in a simpler way.

We find that the issue can be bypassed in the computation of the cusp anomalous dimension, which only sees the $1 / \epsilon$ pole of each HQET diagram. This suggests that complicated terms in individual basis integrals might drop out of the final answer. Having this in mind, we organize the basis in a way such that each basis element only needs to be computed to $O\left(\epsilon^{0}\right)$. Remarkably, the new basis forms a closed differential system up to $O(\epsilon)$ corrections (which are irrelevant for $\left.\Gamma_{\text {cusp }}\right), d \vec{f}-d \mathbf{a}(\epsilon, x) \vec{f}=O(\epsilon)$, where the matrix $\mathbf{a}$ is finite as $\epsilon \rightarrow 0$, and it is nilpotent. To the order needed, the solution is given by multiple polylogarithms.

As we will see presently, only a subset of the integration kernels $\vec{\alpha}$ is relevant for $\Gamma_{\text {cusp }}$. Indeed, the more complicated kernels tend to appear at higher orders in $\epsilon$ of the master integrals. It would be interesting to set up the calculation in a way that makes the simplified function space of the final answer manifest. We think this can be achieved by selecting a basis with manifest infrared and ultraviolet divergence properties, but this goes beyond the scope of this letter.

Results.-Full result for the QED cusp anomalous dimension: We obtain the following result for the fourloop cusp anomalous dimension in QED,

$$
\Gamma_{\text {cusp }}(x, \alpha)=\gamma(\alpha) A(x)+\left(\frac{\alpha}{\pi}\right)^{4} n_{f} B(x)+\mathcal{O}\left(\alpha^{5}\right),
$$

where

$$
\begin{aligned}
\gamma(\alpha)= & \left(\frac{\alpha}{\pi}\right)-\frac{5 n_{f}}{9}\left(\frac{\alpha}{\pi}\right)^{2}+\left(-\frac{n_{f}^{2}}{27}-\frac{55 n_{f}}{48}+n_{f} \zeta_{3}\right)\left(\frac{\alpha}{\pi}\right)^{3} \\
& +\left[n_{f}^{3}\left(-\frac{1}{81}+\frac{2 \zeta_{3}}{27}\right)+n_{f}^{2}\left(\frac{299}{648}+\frac{\pi^{4}}{180}-\frac{10 \zeta_{3}}{9}\right)\right. \\
& \left.+n_{f}\left(\frac{143}{288}+\frac{37 \zeta_{3}}{24}-\frac{5 \zeta_{5}}{2}\right)\right]\left(\frac{\alpha}{\pi}\right)^{4},
\end{aligned}
$$

and

$$
A=-\frac{1+x^{2}}{1-x^{2}} \log x-1
$$

is the one-loop function.

The first term in Eq. (3) comes from propagator-type diagrams [31], while the term $B$, our main new result, comes from the Feynman diagrams shown in Fig. 1. We write it in the following way:

$$
B=\frac{1+x^{2}}{1-x^{2}} B_{1}+\frac{x}{1-x^{2}} B_{2}+\frac{1-x^{2}}{x} B_{3}+B_{4},
$$

which makes the rational dependence on $x$ manifest. The functions $B_{i}$ are given by linear combinations (in $\mathbb{Q}$ ) of multiple polylogarithms of transcendental weight three to seven. We provide them as computer-readable ancillary files. Remarkably, only four of the integration kernels of Eq. (2) appear, namely, $\alpha=\left\{x, 1 \pm x, 1+x^{2}\right\}$.

Previously it was conjectured [10] that $B(x)$ equals

$$
B_{c}(x)=\left(\frac{\pi^{2}}{6}-\frac{\zeta_{3}}{3}-\frac{5 \zeta_{5}}{3}\right) A(x) \approx-0.484 A(x) .
$$

This was found to be inconsistent with the small angle expansion, but curiously it approximately agrees numerically with the exact answer [17]. We can now evaluate our exact result $B(x)-B_{c}(x)$ for any value of $x$, using [32]. Remarkably, we find that the shape of $B(x)$ is very well described by $B_{c}(x)$. It turns out that one can improve the quantitative agreement by adjusting the proportionality constant in Eq. (7). Figures 3(a) and 3(c) show that $-0.458 A(x)$ approximates $B(x)$ within $6 \%$ in a large part of the kinematic regions for Minkowskian angles $\varphi=-i \phi$ and Euclidean angles, respectively, and similarly for the imaginary part in the region $x \in(-1,0)$, as shown in Fig. 3(b). These last two regions can be interpreted as below and above threshold for the production of two massive quarks (see, e.g., [33]). For the real part for $x \in(-1,0)$, the deviation can reach $25 \%$ near $x=-0.2$; see Fig. 3(d). Still, we find it remarkable that the simple one-loop function $A(x)$ captures the main features of the four-loop result. What makes this even more intriguing is that the four terms in Eq. (6) individually have a very different shape, and it is only their sum that resembles the one-loop function.

Let us now expand our novel results in several interesting limits. This serves both as a check and allows us to produce novel results, by computing additional terms.

(1) In the small angle limit $\phi \rightarrow 0$, i.e., $x \rightarrow 1$, we find agreement with known terms up to $\phi^{6}[17,34,35]$. The leading term is $B=\left[\left(5 \pi^{2} / 54\right)+\left(5 \pi^{4} / 108\right)-\right.$ $\left.\left(4 \pi^{2} \zeta_{3} / 9\right)\right] \phi^{2}+\mathcal{O}\left(\phi^{4}\right)$.

(2) In the lightlike limit $x \rightarrow 0$, we find

$$
\begin{aligned}
B= & -\log x\left(\frac{\pi^{2}}{6}-\frac{\zeta_{3}}{3}-\frac{5 \zeta_{5}}{3}\right)+\frac{5 \pi^{2}}{8}-\frac{11 \pi^{4}}{36}+\frac{53 \pi^{6}}{2835} \\
& -\frac{35 \zeta_{3}}{12}-\frac{\pi^{2} \zeta_{3}}{6}-3 \zeta_{3}^{2}+\frac{185 \zeta_{5}}{12}+\mathcal{O}(x) .
\end{aligned}
$$

The first $\log x$ term agrees with the lightlike cusp anomalous dimension [12,13], and the finite part is new.

(3) In the antiparallel lines limit $x \rightarrow-1$, the cusp anomalous dimension is related to the quark-antiquark

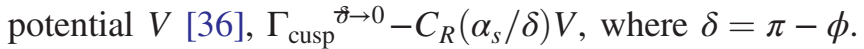
This relation is true up to beta function terms [11], which 
(a)

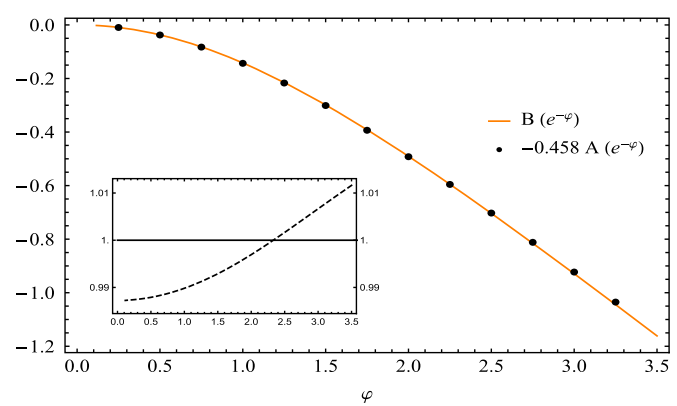

(c)

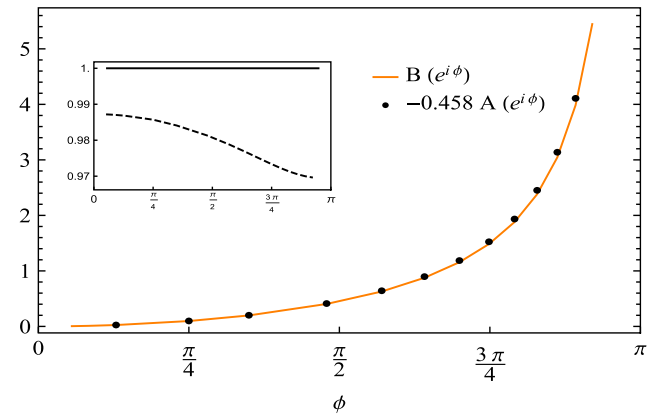

(b)

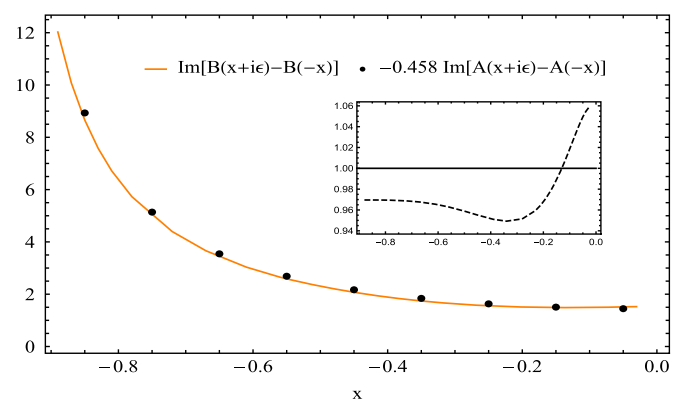

(d)

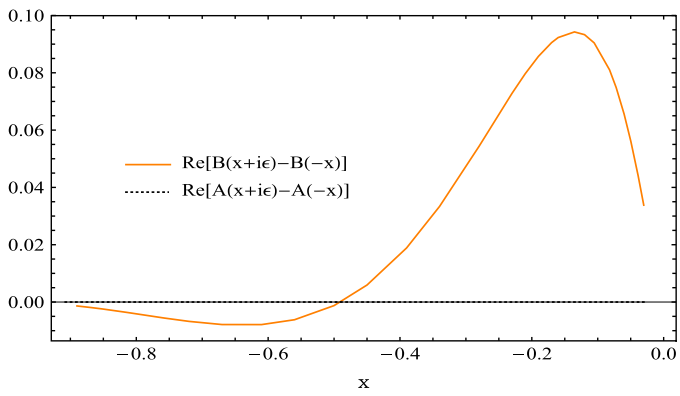

FIG. 3. The shape of the four-loop term $B$ agrees well with the rescaled one-loop function $A$. The dashed line in the insets shows their ratio, which agrees with unity within a few percent, except for (d). The plots show different kinematic regions, for Minkowskian angle $\varphi=-i \phi(\mathrm{a})$, Euclidean angle $\phi(\mathrm{c})$, and for $x \in(-1,0)(\mathrm{b}),(\mathrm{d})$.

do not affect the quartic Casimir term considered here. We find

$$
\begin{aligned}
B= & -\frac{\pi}{\delta}\left(\frac{79 \pi^{2}}{72}-\frac{23 \pi^{4}}{48}+\frac{5 \pi^{6}}{192}+\frac{l_{2} \pi^{2}}{2}+\frac{l_{2} \pi^{4}}{12}\right. \\
& \left.-\frac{l_{2}^{2} \pi^{4}}{4}-\frac{61 \pi^{2} \zeta_{3}}{24}+\frac{21 \pi^{2} \zeta_{3} l_{2}}{4}\right)+\mathcal{O}(\delta),
\end{aligned}
$$

where $l_{2}=\log (2)$. The pole term is in agreement with [37]. It is remarkable that the subleading terms in Eq. (9) start at $\mathcal{O}(\delta)$ only.

Quark-antiquark potential in $\mathcal{N}=4$ SYM: The quarkantiquark potential in $\mathcal{N}=4 \mathrm{SYM}$ is known up to two loops for both the bosonic and supersymmetric Wilson loop [38]. At three loops, it has the following color dependence:

$$
\left.V_{\mathrm{SYM}}\right|_{\alpha_{s}^{3}}=\left(\frac{\alpha_{s}}{\pi}\right)^{3}\left[C_{A}^{3} V_{1}+d_{R} d_{A} /\left(N_{R} C_{R}\right) V_{2}\right]
$$

We can determine $V_{2}$ for bosonic static charges by using a supersymmetric decomposition, as in [14]. Taking into account the known results for the gluon and the fermion quartic Casimir terms [37], we only need the scalar contribution. We obtain the latter from our result $C(x)$ in Eq. (1). [The full formula for $C(x)$ is provided in the Supplemental Material [30]] We find

$$
\begin{aligned}
V_{2}= & 7 \pi^{2}-\frac{47 \pi^{4}}{24}+\frac{413 \pi^{6}}{1440}+\frac{116 \pi^{2} l_{2}}{3}+\frac{3 \pi^{4} l_{2}}{2}+\frac{2}{3} \pi^{4} l_{2}^{2} \\
& -\frac{17}{12} \pi^{2} l_{2}^{4}-34 \pi^{2} \operatorname{Li}_{4}\left(\frac{1}{2}\right)-\frac{89}{4} \pi^{2} \zeta_{3}-14 \pi^{2} l_{2} \zeta_{3} .
\end{aligned}
$$

This constitutes the first nonplanar correction to the quarkantiquark potential in $\mathcal{N}=4 \mathrm{SYM}$. It would be interesting if integrability methods [39] could be extended to this case.

Discussion and conclusions.-We computed the matterdependent quartic Casimir term of the four-loop angledependent cusp anomalous dimension, in a generic gauge theory involving massless matter fields. In particular, this determines the full QED result for this quantity and includes for the first time contributions from light-by-light scattering diagrams.

Our calculation revealed new structures compared to previous three-loop results: the answer contains four as opposed to previously two rational structures. The function space is given by iterated integrals, and compared to three loops, there is one new integration kernel, namely, $d \log \left(1+x^{2}\right)$, in addition to $d \log x, d \log (1-x)$, and $d \log (1+x)$. This relative simplicity is remarkable given that intermediate steps (and higher-order terms in the dimensional regulator) contain further integration kernels, cf. Eq. (2). This hints at better approaches that avoid the complicated intermediate terms. The new kernel has singularities at $x= \pm i$, which correspond to $\phi= \pm 90^{\circ}$. We remark that $\Gamma_{\text {cusp }}$ is smooth at this location. This is 
reminiscent of what has been observed for massive form factors in [33].

The information on the function space is valuable input for bootstrap approaches [40]. In particular, our result implies constraints (via collinear limits) on the multileg soft anomalous dimension matrix for massive particles, which currently is known at two loops only [8].

We analyzed the novel four-loop result numerically and found that, surprisingly, it is described within a few percent by a rescaled one-loop function (and up to $25 \%$ for the real part in the above threshold region). Clearly this could be improved even further by using some of the known limiting behavior, as in [41], for example. It would be interesting to understand why the approximation works so well here, in view of other problems where fully analytic results are not yet known, such as scattering processes with many mass scales.

To obtain all color contributions of the complete QCD cusp anomalous dimension at four loops, only two further contributions are needed [18]. The first one is the planar limit, which is conceptually easier compared to our calculation. The HQET integrals we computed should cover a large part of the integrals needed and are available upon request from the authors. The second one is the gluonic quartic Casimir term. Thanks to our calculation of the scalar terms, the latter can equivalently be obtained from the nonplanar $\mathcal{N}=4 \mathrm{SYM}$ contribution.

This research received funding from the European Research Council (ERC) under the European Union's Horizon 2020 Research and Innovation Programme (Grant Agreement No. 725110), Novel structures in scattering amplitudes. R. B. was supported by the Deutsche Forschungsgemeinschaft (DFG) under Grant No. 396021762-TRR 257.

*robin.brueser@uni-siegen.de

†dlapa@mpp.mpg.de

thenn@mpp.mpg.de

§kyan@mpp.mpg.de

[1] S. Weinberg, Phys. Rev. 140, B516 (1965); G. Sterman, Nucl. Phys. B281, 310 (1987); J. C. Collins, D. E. Soper, and G. F. Sterman, Adv. Ser. Dir. High Energy Phys. 5, 1 (1989).

[2] G. Korchemsky and A. Radyushkin, Phys. Lett. B 279, 359 (1992); L. J. Dixon, L. Magnea, and G. F. Sterman, J. High Energy Phys. 08 (2008) 022.

[3] N. Arkani-Hamed, J. L. Bourjaily, F. Cachazo, and J. Trnka, J. High Energy Phys. 06 (2012) 125; C. Gnendiger et al., Eur. Phys. J. C 77, 471 (2017); C. Anastasiou and G. Sterman, J. High Energy Phys. 07 (2019) 056; H. Hannesdottir and M. D. Schwartz, arXiv:1906.03271.

[4] J. Drummond, J. Henn, G. Korchemsky, and E. Sokatchev, Nucl. Phys. B826, 337 (2010); D. Chicherin, J. M. Henn, and E. Sokatchev, Phys. Rev. Lett. 121, 021602 (2018).
[5] Z. Bern, L. J. Dixon, and V. A. Smirnov, Phys. Rev. D 72, 085001 (2005).

[6] O. Almelid, C. Duhr, and E. Gardi, Phys. Rev. Lett. 117, 172002 (2016).

[7] N. Beisert, B. Eden, and M. Staudacher, J. Stat. Mech. (2007) P01021.

[8] A. Mitov, G. F. Sterman, and I. Sung, Phys. Rev. D 79, 094015 (2009); A. Ferroglia, M. Neubert, B. D. Pecjak, and L. L. Yang, Phys. Rev. Lett. 103, 201601 (2009); Y.-T. Chien, M. D. Schwartz, D. Simmons-Duffin, and I. W. Stewart, Phys. Rev. D 85, 045010 (2012).

[9] G. Korchemsky and A. Radyushkin, Nucl. Phys. B283, 342 (1987).

[10] A. Grozin, J. M. Henn, G. P. Korchemsky, and P. Marquard, Phys. Rev. Lett. 114, 062006 (2015).

[11] A. Grozin, J. M. Henn, G. P. Korchemsky, and P. Marquard, J. High Energy Phys. 01 (2016) 140.

[12] R. N. Lee, A. V. Smirnov, V. A. Smirnov, and M. Steinhauser, J. High Energy Phys. 02 (2019) 172.

[13] J. M. Henn, T. Peraro, M. Stahlhofen, and P. Wasser, Phys. Rev. Lett. 122, 201602 (2019).

[14] J. M. Henn, G. P. Korchemsky, and B. Mistlberger, J. High Energy Phys. 04 (2020) 018.

[15] T. Huber, A. von Manteuffel, E. Panzer, R. M. Schabinger, and G. Yang, Phys. Lett. B 807, 135543 (2020).

[16] A. von Manteuffel, E. Panzer, and R. M. Schabinger, Phys. Rev. Lett. 124, 162001 (2020).

[17] A. Grozin, J. Henn, and M. Stahlhofen, J. High Energy Phys. 10 (2017) 052.

[18] R. Brüser, A. Grozin, J. M. Henn, and M. Stahlhofen, J. High Energy Phys. 05 (2019) 186.

[19] T. van Ritbergen, A. Schellekens, and J. Vermaseren, Int. J. Mod. Phys. A 14, 41 (1999); F. Herzog, B. Ruijl, T. Ueda, J. Vermaseren, and A. Vogt, J. High Energy Phys. 02 (2017) 090.

[20] J. M. Henn, Phys. Rev. Lett. 110, 251601 (2013).

[21] A. V. Kotikov, Phys. Lett. B 254, 158 (1991); Z. Bern, L. J. Dixon, and D. A. Kosower, Nucl. Phys. B412, 751 (1994); E. Remiddi, Nuovo Cimento A 110, 1435 (1997); T. Gehrmann and E. Remiddi, Nucl. Phys. B580, 485 (2000).

[22] A. Smirnov and F. Chuharev, Comput. Phys. Commun. 247, 106877 (2020).

[23] R. N. Lee, J. Phys. Conf. Ser. 523, 012059 (2014).

[24] T. Peraro, J. High Energy Phys. 12 (2016) 030.

[25] P. S. Wang, M. J. T. Guy, and J. H. Davenport, SIGSAM Bulletin 16, 2 (1982); A. von Manteuffel and R. M. Schabinger, Phys. Lett. B 744, 101 (2015).

[26] C. Dlapa, J. Henn, and K. Yan, J. High Energy Phys. 05 (2020) 025.

[27] R. N. Lee, J. High Energy Phys. 04 (2015) 108.

[28] O. Gituliar and V. Magerya, Comput. Phys. Commun. 219, 329 (2017).

[29] C. Duhr, Annu. Rev. Nucl. Part. Sci. 69, 15 (2019).

[30] See Supplemental Material at http://link.aps.org/ supplemental/10.1103/PhysRevLett.126.021601 for information on the differential equations for the 10-propagator sector shown in Fig. 2, and analytic expressions for the functions $B_{i}$ defined in Eq. (6) for the term $B$, and for $C_{i}$ defined in the same way for the term $C$. 
[31] J. Gracey, Phys. Lett. B 322, 141 (1994); M. Beneke and V. M. Braun, Nucl. Phys. B454, 253 (1995).

[32] C. W. Bauer, A. Frink, and R. Kreckel, J. Symb. Comput. 33, 1 (2002); J. Vollinga and S. Weinzierl, Comput. Phys. Commun. 167, 177 (2005).

[33] J. Henn, A. V. Smirnov, V. A. Smirnov, and M. Steinhauser, J. High Energy Phys. 01 (2017) 074.

[34] A. Grozin, J. High Energy Phys. 06 (2018) 073; 01 (2019) 134(A).

[35] The sign of $\alpha^{4} n_{l}^{2}$ in Eq. (4.2) of [34] should be reversed.

[36] W. Kilian, T. Mannel, and T. Ohl, Phys. Lett. B 304, 311 (1993).

[37] R. N. Lee, A. V. Smirnov, V. A. Smirnov, and M. Steinhauser, Phys. Rev. D 94, 054029 (2016).
[38] N. Drukker, D. J. Gross, and H. Ooguri, Phys. Rev. D 60, 125006 (1999); J. K Erickson, G. W. Semenoff, R. J. Szabo, and K. Zarembo, Phys. Rev. D 61, 105006 (2000); A. Pineda, Phys. Rev. D 77, 021701(R) (2008); N. Drukker and V. Forini, J. High Energy Phys. 06 (2011) 131; M. Prausa and M. Steinhauser, Phys. Rev. D 88, 025029 (2013).

[39] D. Correa, J. Maldacena, and A. Sever, J. High Energy Phys. 08 (2012) 134; N. Drukker, J. High Energy Phys. 10 (2013) 135; N. Gromov and F. Levkovich-Maslyuk, J. High Energy Phys. 12 (2016) 122.

[40] O. Almelid, C. Duhr, E. Gardi, A. McLeod, and C. D. White, J. High Energy Phys. 09 (2017) 073.

[41] J. Davies, R. Gröber, A. Maier, T. Rauh, and M. Steinhauser, Proc. Sci. RADCOR2019 (2019) 079. 